\title{
Erythema exsudativum multiforme-artiges Mykid unter systemischer Therapie einer Trichophyton interdigitale-Infektion ${ }^{1}$
}

\author{
Erythema Exsudativum Multiforme-Like Mycid During Systemic Antimycotic Therapy of a Trichophyton \\ Interdigitale-Infection
}

Autoren

Institute
L. Kowalzick ${ }^{1}$, L. Eickenscheidt ${ }^{1}$, B. Schell' ${ }^{1}$, H. Ziegler ${ }^{1}$, F. Weiße ${ }^{2}$, J.-M. Pönnighaus ${ }^{1}$, R. A. Herbst ${ }^{2}$

Klinik für Hautkrankheiten und Allergologie (Chefarzt: Prof. Dr. med. habil. L. Kowalzick),

HELIOS Vogtland-Klinikum Plauen GmbH (Ärztl. Direktor: Prof. Dr. med. habil. L. Kowalzick)

${ }^{2}$ Klinik für Hautkrankheiten und Allergologie (Chefarzt: Prof. Dr. med. R. A. Herbst),

HELIOS Klinikum Erfurt GmbH (Ärztl. Direktor: Prof. Dr. med. D. Esser)

\section{Bibliografie}

DOI http://dx.doi.org/

10.1055/s-0029-1215040

Online-Publikation: 18. 8. 2009

Akt Dermatol 2010; 36:

136-138 @ Georg Thieme

Verlag KG Stuttgart · New York ISSN 0340-2541

\section{Korrespondenzadresse}

Prof. Dr. med. habil.

Lutz Kowalzick

Klinik für Hautkrankheiten und Allergologie

HELIOS Vogtland-Klinikum

Plauen $\mathrm{GmbH}$

Postfach 100153

08505 Plauen

lutz.kowalzick@

helios-kliniken.de

\section{Zusammenfassung \\ $\nabla$}

Wir berichten über einen 22-jährigen Patienten, der kurz nach Initiierung einer systemischen antimykotischen Therapie mit Terbinafin wegen einer mykologisch gesicherten Tinea, verursacht durch Trichophyton interdigitale (Teleomorphe: Arthroderma benhamiae) am linken Handrücken, dann an beiden Händen, Unterarmen, distalen Oberarmen und Knien, z.T. konfluierende Erythema exsudativum multiforme-artige Hautveränderungen mit sehr starkem Juckreiz entwickelte. Histologisch ließ sich eine Ekzemreaktion nachweisen,

\section{Einleitung}

Das Konzept der „Id-Reaktion“ ist alt, und Kriterien für die Diagnosestellung wurden bereits 1921 für das Mykid bzw. Dermatophytid oder Trichophytid formuliert [7]. Auch Bakteride und Viruside wurden postuliert [5]. Der Begriff bezeichnet hypererge, morphologisch unterschiedliche Reaktionen der Haut auf mikrobielle Antigene an vom Infektionsort weiter entfernten Lokalisationen, die nach Therapie der Grunderkrankung abheilen $[1,2,4,5]$. Wir berichten hier über einen Patienten mit einer Tinea und mit einem Erythema exsudativum multiforme-artigen Mykid und das erfolgreiche Ansprechen auf eine spezifische topische und systemische Therapie.

\section{Kasuistik}

Wir berichten über einen 22-jährigen Patienten, der von einem peripheren Krankenhaus in unserer Notfallambulanz vorgestellt wurde. Er war etwa zwei Wochen zuvor an einer umschriebenen, randbetont geröteten und schuppenden

${ }^{1}$ Herrn Prof. Dr. med. T. Nasemann, Bernried, zu seinem 86. Geburtstag gewidmet. die gut mit einem Mykid vereinbar war. Nach Wiederaufnahme der systemischen antimykotischen Therapie und unter einer systemischen Prednisolon-Stoßtherapie über 5 Tage bildeten sich sowohl die Tinea als auch das Mykid rasch zurück. Diese Kasuistik soll demonstrieren, dass das Auftreten eines Mykids gerade nach Beginn einer spezifischen antimykotischen Therapie einer Mykose möglich ist und keineswegs zum Absetzen dieser Medikation, sondern zu einer konsequenten Fortsetzung der Therapie der Grundkrankheit führen sollte.

Hautveränderung im Bereich des linken ulnaren Handrückens erkrankt. Vom Hausarzt erhielt der Patient für einige Tage ein topisches Kortikosteroid, worunter der Herd sich weiter zentrifugal ausdehnte. Im Haushalt des Patienten wurden unter anderem Kaninchen und Meerschweinchen gehalten. Der niedergelassene Dermatologe fertigte ein mykologisches Nativpräparat an, in dem sich reichliche Pilzstrukturen fanden. Daraufhin wurde eine systemische Therapie mit Terbinafin in einer Dosis von $250 \mathrm{mg}$ tgl. per os eingeleitet. Binnen 24 Stunden kam es dann schlagartig zur Aussaat neuer geröteter und stark juckender Hautveränderungen an beiden Handrücken, Unterarmen, distalen Oberarmen und Knien.

Es fand sich im Bereich des linken ulnaren Handrückens ein ca. $6 \times 4,5 \mathrm{~cm}$ durchmessendes, randbetont coloretteartig schuppendes, gering exsudatives, erythematöses Areal mit zentral einzelnen Pusteln. Im Bereich beider Handrücken, beider Unterarme und beider distaler Oberarme fanden sich insbesondere distal flächig konfluierende, wenige, bis ca. $1 \mathrm{~cm}$ durchmessende, randbetont elevierte, erythematöse Papeln mit angedeuteter Kokardenbildung ( $\boldsymbol{\otimes}$ Abb. 1).

Wir führten eine Probebiopsie aus dem Bereich der neu aufgetretenen papulösen Hautveränderungen durch. Es fand sich eine deutlich verbrei- 


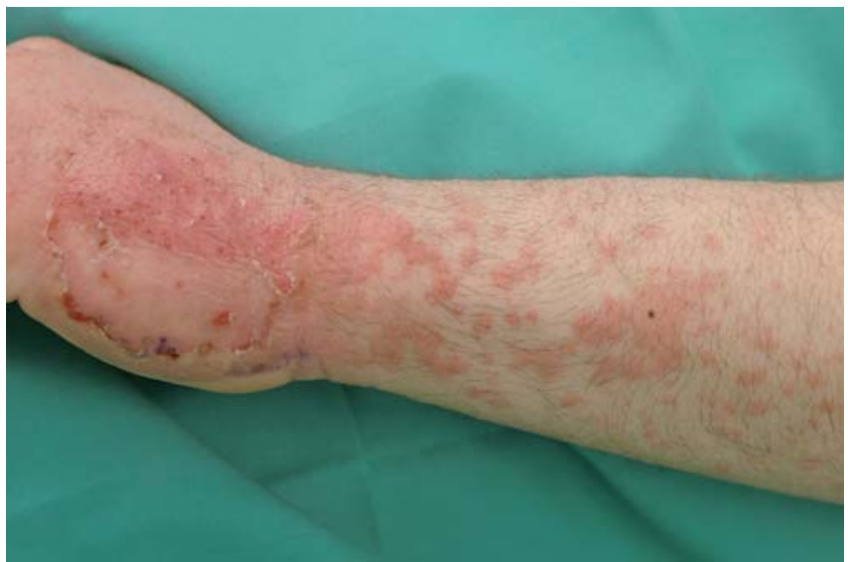

Abb. 1 Linke Hand und Unterarm eines 22-jährigen Patienten mit einer Tinea, bedingt durch Trichophyton interdigitale (Arthroderma benhamiae), am ulnaren proximalen Handrücken mit kranzförmiger Schuppung, gering erosivem Erythem und zentralen Pusteln (links) und teils kokardenförmigen (rechts) infiltrierten, z. T. konfluierenden multiformen Erythemen.

terte Epidermis mit Orthokeratose und massiven Spongiosezeichen mit z.T. großen intradermalen Vesikeln. Im angrenzenden Korium zeigte sich ein vorwiegend perivasal angeordnetes lymphohistiozytäres Infiltrat mit epidermaler Exozytose. Eosinophile fanden sich nicht, und nur vereinzelt neutrophile Granulozyten (৫ Abb. 2).

In der PAS-Färbung ließen sich keinerlei Pilzelemente nachweisen ( $\bullet$ Abb. 2). Insgesamt zeigte sich das histologische Bild eines subakuten Ekzems, das gut mit einem Mykid vereinbar war [3]. Mehrere mykologische Nativpräparate $(\mathrm{KOH})$ aus dem Tinea-Bereich waren dagegen massiv positiv. In der mykologischen Kultur ließ sich das Wachstum des Dermatophyten Trichophyton interdigitale (vormals: Trichophyton rubrum) nachweisen. Die MALDI-TOF-Massenspektroskopie-Analyse ermöglichte die Identifizierung des perfekten Stadiums (Teleomorphe) Arthroderma benhamiae, eines zoophilen Dermatophyten, der besonders durch Nagetiere übertragen wird [6].

Wir diagnostizierten klinisch ein Mykid und setzten die mittlerweile 48 Stunden unterbrochene systemische Terbinafin-Therapie $(250 \mathrm{mg} /$ die) wieder fort. Wir behandelten lokal im Bereich der Tinea mit $1 \%$ Batrafen-Creme ${ }^{\circledR} 2 \times$ tgl. und gaben systemisch $80 \mathrm{mg}$ (ca. $1 \mathrm{mg} / \mathrm{kg}$ Körpergewicht) Prednisolon p. o. als Stoß über 5 Tage. Die exanthematischen multiformen Veränderungen behandelten wir topisch mit Bethametasonvalerat $0,1 \%$ in Unguentum leniens. Hierunter kam es zu einem raschen Abblassen und Abflachen der infiltrierten Erytheme, sodass der Patient nach 6 Tagen mit der Maßgabe, die systemische und lokale antimykotische Therapie noch mindestens 2 Wochen fortzusetzen und seine Nagetiere tierärztlich untersuchen und ggf. ebenfalls behandeln zu lassen, weitgehend abgeheilt in die weitere ambulante dermatologische Behandlung entlassen werden konnte.

\section{Diskussion}

$\nabla$

Als Mykid werden bei hypererger Reaktionslage im Laufe einer Dermatophytose auftretende, auch fern vom Tineaherd auftretende Hauterscheinungen, in denen keine Pilze nachweisbar sind, bezeichnet. Vermutet wird eine Resorption von Pilzantigenen auch fern vom Herd. Klinisch handelt es sich um symmetri-

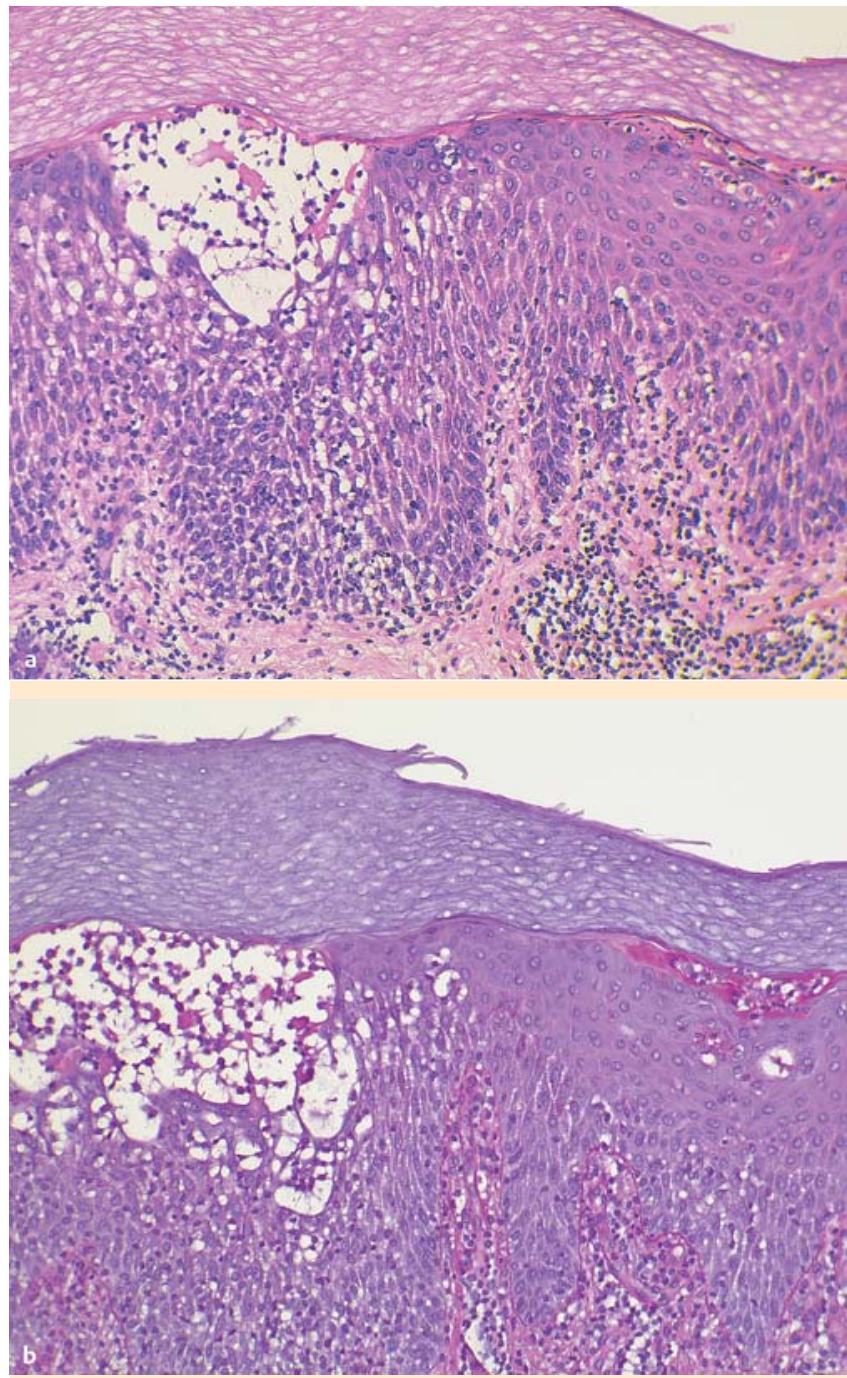

Abb. 2 a, b Histologie aus den Erythema exsudativum multiforme-artigen Hautveränderungen: Deutlich verbreiterte Epidermis mit Orthokeratose und massiven Spongiosezeichen mit z. T. großen intradermalen Vesikeln. Im angrenzenden Korium zeigt sich ein vorwiegend perivasal angeordnetes lymphohistiozytäres Infiltrat mit epidermaler Exozytose (a). Eine parallele PAS-Färbung zeigte keinerlei Pilzelemente (b). Diagnose: Subakutes Ekzem, gut mit einem Mykid vereinbar.

sche, dyshidrosiforme Eruptionen an Händen und Füßen oder um lichenoide, nodöse und multiforme Erytheme in Extremitäten-betonten Lokalisationen [4,5]. Letztere Konstellation war in unserem Fall klassisch gegeben und erlaubte die rasche klinische Diagnose, die auch durch die später vorliegenden histologischen und mykologischen Befunde gestützt wurde. Es wird beobachtet, dass Mykide insbesondere auch während einer intensiven Behandlung der manifesten Mykose auftreten, möglicherweise infolge einer verstärkten Antigenresorption bei Abtötung der Erreger $[4,9]$. Auch dies trifft auf unseren Fall zu, bei dem die EEMartigen Hautveränderungen erst unmittelbar nach Beginn einer systemischen antimykotischen Therapie auftraten.

Das Konzept der „Id-Reaktion“ ist alt, und Kriterien für die Diagnosestellungen wurden bereits 1921 für das Mykid bzw. Dermatophytid oder Trichophytid formuliert. In vielen Fällen wurde ein stark positiver Intrakutantest mit Trichophytin gefunden, wie er bis vor einigen Jahren im Multitest für Recall-Antigene enthalten war [7]. Im Einzelfall wurde auch über eine Exazerbation des 
Exanthems nach Trichophytin-Testung berichtet [8]. Auch der Nachweis von Pilzstrukturen im Blut wurde ursprünglich beschrieben, dieses Postulat aber später wieder verlassen [7].

Vor einigen Jahren wurde ein unserem ähnlicher Fall aus Italien berichtet, bei dem im Verlauf einer im Gesicht lokalisierten Tinea, gleichfalls bedingt durch Trichophyton interdigitale sive mentagrophytes, eine exanthematische Aussaat von erythematös-ödematösen targetoiden Hautveränderungen auftrat, die unter systemischer antimykotischer Therapie der Tinea mit Terbinafin binnen 7 Tagen abheilte [2].

Auch im gegenwärtigen Fall bildete sich das Mykid unter der generell empfohlenen konsequenten antimykotischen (Weiter-) Therapie $[1,4,5,9]$ zurück. In unserem Kasus war die systemische antimykotische Therapie zunächst unterbrochen worden, da zuerst eine allergische Reaktion auf das neu eingenommene Terbinafin vermutet wurde. Der Verlauf zeigt aber, dass in Kenntnis der Klinik und des Ursachenzusammenhanges des Mykids nur eine konsequente Weiterbehandlung bzw. Wiederaufnahme der Therapie kausal wirksam und erfolgreich sein wird.

\section{Danksagung}

$\nabla$

Wir danken Herrn Prof. Dr. P. Nenoff, Laboratorium für medizinische Mikrobiologie, Bakteriologie, Mykologie, Virologie \& Infektionsserologie, Espenhain, für die Mitbefundung der mykologischen Kultur und Herrn Dr. M. Erhard, Potsdam, für die massenspektrokopische Identifizierung der Teleomorphe des Erregers.

\section{Abstract}

\section{Erythema Exsudativum Multiforme-Like Mycid During Systemic Antimycotic Therapy of a Trichophyton Inter- digitale-Infection}

We report on a 22-years old male caucasian patient who developed strongly itching erythema exsudativum multiforme-like skin lesions on both his hands, forearms, distal upper arms and knees, shortly after beginning of a systemic antimycotic therapy with terbinafine because of a mycological proven tinea of the back of his left hand caused by Trichophyton interdigitale (teleomorph Arthroderma benhamiae). Histology showed an eczematous dermatitis which supports well the clinical diagnosis of a mycid. After restarting the systemic antimycotic treatment and the administration of a 5 days bolus prednisolone therapy the tinea as well as the mycid improved quickly. This case demonstrates that the appearance of a mycid, especially after the start of a specific antifungal therapy should not lead to a stop of this medication but a consequent continuation of the treatment of the underlying disease.

\section{Literatur}

1 Altmeyer P. Therapielexikon der Dermatologie und Allergologie. 2. Auflage. Heidelberg: Springer, 2005: 410 und 604

2 Atzori L, Pau M, Aste M. Erythema multiforme ID reaction in atypical dermatophytosis: a case report. J Eur Acad Dermatol Venereol 2003; 17: $699-701$

3 Cohen LM, Skopicki DK, Harrist TJ, Clark WH. Noninfectious vesiculobullous and vesiculopustular diseases. In: Elder D, Elenistas R, Jaworsky C, Johnson B (Hrsg). Lever's histopathology of the skin. 8. Auflage. Philadelphia: Lippincott-Raven, 1997: 214

4 Korting HC. Mykosen. In: Braun-Falco O, Plewig GW, Wolff $\mathrm{HH}$ et al. (Hrsg). Dermatologie und Venerologie, 5. Auflage. Heidelberg: Springer, 2005: $191-192$

5 Nasemann T, Sauerbrey W. Lehrbuch der Hautkrankheiten und venerischen Infektionen, 2. Auflage. Heidelberg: Springer, 1977: 45, 80, $84-$ 85

6 Nenoff P, Herrmann J, Gläser Y. Trichophyton mentagrophytes sive interdigitale? A dermatophyte in the course of time. J Dt Dermatol Ges 2007; 5: 198-202

7 Simons RD. Über die Dyshidrose bei der Tinea pedis. Das Mykid-Konzept im Jahre 1965. Arch Klin Exp Dermatol 1966; 227: 550-560

8 Steigleder GK. Mykose mit varioliformen nekrotisierendem Mykid nach Trichophytin-Injektion. Hautarzt 1953; 4: 35-36

9 Steigleder GK. Dermatologie und Venerologie. 3. Auflage. Stuttgart: Thieme, 1979: 279-280 